\title{
Growth inhibition and metabolomic analysis of Xanthomonas oryzae pv. oryzae treated with resveratrol
}

Huai-Zhi Luo ${ }^{1,2 \dagger}$, Ying Guan ${ }^{3+}$, Rui Yang ${ }^{1 \dagger}$, Guo-Liang Qian ${ }^{4}$, Xian-Hui Yang ${ }^{5^{*}}$, Jun-Song Wang ${ }^{1 *}$ and Ai-Qun Jia ${ }^{1,2^{*}}$ (D)

\begin{abstract}
Background: Xanthomonas oryzae pv. oryzae (Xoo) can cause destructive bacterial blight in rice. As an antibacterial, resveratrol may inhibit $X_{00}$ growth. This study focused on the potential structural-activity relationship of resveratrol and its derivatives against Xoo growth, and ${ }^{1} \mathrm{H}$-NMR-based metabolomic analysis was applied to investigate the global metabolite changes in Xoo after resveratrol treatment.

Results: Resveratrol showed the strongest inhibitory effects on Xoo growth compared with its derivatives, which lacked double bonds (compounds 4-6) or hydroxyls were substituted with methoxyls (compounds 7-9). The $I C_{50}$ of resveratrol against $X_{0 O}$ growth was $11.67 \pm 0.58 \mu \mathrm{g} / \mathrm{mL}$. Results indicated that the double bond of resveratrol contributed to its inhibitory effects on Xoo growth, and hydroxyls were vital for this inhibition. Interestingly, resveratrol also significantly inhibited $X_{0 O}$ flagellum growth. Based on ${ }^{1} \mathrm{H}$-NMR global metabolic analysis, a total of 30 Xoo metabolites were identified, the changes in the metabolic profile indicated that resveratrol could cause oxidative stress as well as disturb energy, purine, amino acid, and NAD ${ }^{+}$metabolism in Xoo, resulting in the observed inhibitory effects on growth.

Conclusions: This study showed that the double bond of resveratrol contributed to its inhibitory effects on Xoo growth, and hydroxyls were also the important active groups. Resveratrol could cause oxidative stress of Xoo cells, and disturb the metabolism of energy, purine, amino acid and NAD +, thus inhibit Xoo growth.
\end{abstract}

Keywords: Xanthomonas oryzae pv. Oryzae, Resveratrol, Flagella, Structure-activity relationship, Global metabolic analysis

\section{Background}

Xanthomonas is an important genus of gram-negative pathogenic bacteria, and can infect approximately 350 different plants [1]. Xanthomonas oryzae pv. oryzae $(\mathrm{Xoo})$ is one of the most important bacterial pathogens in rice, and can cause destructive bacterial blight $(\mathrm{BB})$ in many regions world-wide [2]. $\mathrm{BB}$ is an economically

\footnotetext{
*Correspondence: yxh966@126.com; wang.junsong@gmail.com; jiaaiqun@gmail.com

${ }^{+}$Huai-Zhi Luo, Ying Guan and Rui Yang contributed equally to this work.

${ }^{5}$ School of Science, Hainan University, Haikou 570228, China

${ }^{1}$ School of Environmental and Biological Engineering, Nanjing University of Science and Technology, Nanjing 210094, China

Full list of author information is available at the end of the article
}

significant and highly devastating disease of rice, and has been reported in Asia, Africa, Australia and Latin America [1]. BB can cause at least $10 \%$ yield loss in susceptible rice varieties, and up to $60 \%$ yield loss during severe epidemics [3]. Bactericides and antibacterial agents have become indispensable tools in the control of $\mathrm{BB}$, and include the most commonly used copper-based bactericides and zinc thiazole [3]. However, these bactericide agents also exhibit many shortcomings, including poor efficacy, influence on nontarget organisms, high phytotoxicity, environmentally unfriendly, and bactericide resistance, so their use is relatively unsustainble [4]. Many antibiotics, such as streptomycin, have also been used to control BB [5], with benzylpenicillin, ampicillin, kanamycin, chloramphenicol, and sinobionics

(c) The Author(s). 2020 Open Access This article is licensed under a Creative Commons Attribution 4.0 International License, which permits use, sharing, adaptation, distribution and reproduction in any medium or format, as long as you give appropriate credit to the original author(s) and the source, provide a link to the Creative Commons licence, and indicate if changes were made. The images or other third party material in this article are included in the article's Creative Commons licence, unless indicated otherwise in a credit line to the material. If material is not included in the article's Creative Commons licence and your intended use is not permitted by statutory regulation or exceeds the permitted use, you will need to obtain permission directly from the copyright holder. To view a copy of this licence, visit http://creativecommons.org/licenses/by/4.0/ The Creative Commons Public Domain Dedication waiver (http://creativecommons.org/publicdomain/zero/1.0/) applies to the data made available in this article, unless otherwise stated in a credit line to the data. 
reported to inhibit Xoo growth [6]. However, over use and abuse of these antibiotic resulted in increasing bacterial resistance. Thus, development of new antibacterial agents for the control of $\mathrm{BB}$ is urgently required. Recently, several natural products have been reported to show antibacterial activity against $X o o$, including peptides, graphene oxide, actinomyces, Adathoda vasica leaf extract, and Datura metel leaf extract [7].

Resveratrol, as a "model stilbene" and an important phytoalexin, is isolated from grapes, berries, peanuts, pines, and Polygonum cuspidatum Sieb [8]. Additionally, resveratrol can be synthesized easily with a high yield, which makes it possible to obtain large scale in low cost [9]. Resveratrol exhibits antioxidant, antiviral, anti-inflammatory, anti-fungal, and anticancer bioactivities and is a known quorum sensing inhibitor (QSI) that can inhibit Pseudomonas aeruginosa virulence [10]. Additionally, resveratrol can be used as a potent antibacterial to inhibit the growth of Xanthomonas [11] and was rescreened in the current study.

To the best of our knowledge, the main active groups of resveratrol, as well as its effects on molecular metabolic profiles and the potential inhibition mechanism against Xanthomonas, remain unknown. With the development of omics technologies, metabolomic has been applied to study metabolic profiles and molecular mechanisms. In this research, we investigated the potential structureactivity relationship of resveratrol and its derivatives and their inhibitory effects on Xoo growth. The metabolic changes in Xoo and underlying inhibition mechanism were also evaluated after treatment with resveratrol using ${ }^{1}$ H-NMR-based metabolomics [10]. Results indicated that the double bond of resveratrol contributed to its inhibition of Xoo growth, with hydroxyls found to be the vital active group. Furthermore, our results suggested that resveratrol could disturb energy, purine, amino acid, and $\mathrm{NAD}^{+}$metabolism in Xoo cells, resulting in the observed inhibitory effects on growth.

\section{Results}

\section{Structural identification of three Stilbenoids (1-3) and} derivatives (4-9)

The chemical structures of three stilbenoids (1-3) and their derivatives (4-9), di-hydro-resveratrol (4), di-hydro-oxyresveratrol (5), di-hydro-piceatannol (6), tri-methyl-resveratrol (7), tetra-methyl-oxyresveratrol (8), and tetra-methyl-piceatannol (9), were shown in Fig. 1. The ${ }^{1} \mathrm{H}$ - and ${ }^{13} \mathrm{C}-\mathrm{NMR}$ chemical shifts of the six derivatives (4-9) were shown in Table S1.

\section{Antibacterial activity of compounds (1-9) against Xoo}

As shown in Fig. 2, compounds (1-6) exhibited antibacterial activity against Xoo, whereas compounds (7-9) had no effects on the growth of Xoo (data not shown). Dramatically, resveratrol (1) showed the strongest antibacterial activity against Xoo $\left(\mathrm{IC}_{50} 11.67 \pm 0.58 \mu \mathrm{g} / \mathrm{mL}\right)$ (Table 1), and at $5 \mu \mathrm{g} / \mathrm{mL}, 25 \mu \mathrm{g} / \mathrm{mL}$, and $100 \mu \mathrm{g} / \mathrm{mL}$, the inhibiting percentage on Xoo growth was $24.66 \pm 1.79,75.84 \pm 3.14$, and $90.49 \pm 0.28$, respectively. For compounds (2-6), the inhibiting percentages on Xoo growth at $100 \mu \mathrm{g} / \mathrm{mL}$ were $89.39 \pm 0.43,78.89 \pm 0.80,82.76 \pm 1.02,54.30 \pm 6.05$, and $58.51 \pm 3.11$, respectively, and at $5 \mu \mathrm{g} / \mathrm{mL}$ were $16.27 \pm 1.06$, $23.96 \pm 3.15,25.24 \pm 7.31,18.51 \pm 2.10$, and $12.38 \pm 2.25$, respectively. As shown in Table 1 , the $\mathrm{IC}_{50}$ values of compounds (2-6) on Xoo growth were $19.00 \pm 1.00,27.00 \pm$ $3.61,36.27 \pm 3.75,123.53 \pm 7.66$, and $115.46 \pm 7.93 \mu \mathrm{g} / \mathrm{mL}$, respectively.

\section{Effects of resveratrol on Xoo flagella}

Bacterial flagella are important virulence factors for pathogenesis of animals and plants, and flagella-driven chemotaxis plays an important role in the early interaction of host plants in some plant-pathogen systems [12]. Xoo harbors a single polar flagellum for motility, and the function of flagella allows bacteria to move away from hazards to favorable conditions by responding to chemical signals [13]. So we investigated whether resveratrol was an inhibitor to influence the Xoo flagella. As shown in Fig. 3, flagella were detected on the surface of Xoo in the blank (Fig. 3a) and DMSO control groups (Fig. $3 \mathrm{~b}$ ), but not in the resveratrol treatment group (Fig. 3c).

\section{Metabolite identification and multivariate statistical analyses}

Typical $500 \mathrm{MHz}$ CPMG ${ }^{1} \mathrm{H}-\mathrm{NMR}$ spectra for the resveratrol-treated ( $\mathrm{T}$-group) and control groups (Cgroup) are shown in Fig. 4, with a total of 30 metabolites assigned. Detailed information on the 30 metabolites is shown in Table 2. The STOCSY technique, which computes correlation among the intensities of all peaks in a matrix, was used for the assignment of metabolites, such as glutamate, succinate, tyrosine, and histidine (Fig. 5).

Principal component analysis (PCA) was first used to obtain an overview of variation between the C-group and $\mathrm{T}$ group. Quite significant overlap was observed between the PCA score plot of two groups in Fig. 6. OSC-PLS-DA, a supervised pattern recognition technique, was used to identify the metabolic differences between the two groups. In the OSC-PLS-DA score plot (Fig. 7a), the T-group was significantly separated from the C-group. The corresponding Splot (Fig. 7d) and the color-coded loading plots (Fig. 7b,c) were used to identify the contributions of variables between the groups. In addition, changes in metabolites were directly visualized as fold-changes in these plots, and were color-coded according to the differences of $p$-values between the groups (Fig. 8). Compared with the C-group, cholate, succinate, taurine, $\mathrm{NAD}^{+}, \mathrm{NADP}^{+}, \mathrm{IMP}, \mathrm{AMP}$, and formate significantly increased, whereas valine, suberate, lysine, glutamate, 2-aminoadipate, glutamine, glutathione, 
<smiles>[R][R]c1c(/C=C/c2cc(O)cc(O)c2)ccc(O)c1[R]</smiles><smiles>[R][R]</smiles><smiles>[R][R]Oc1ccc(/C=C/c2cc(OC)cc(OC)c2)c([R])c1[R1]</smiles>

Fig. 1 Chemical structures of compounds (1-9)

choline, glycine, uridine, and fumarate markedly decreased in the T-group. The assigned metabolites, their fold change values, and $p$-values are shown in Table 2.

\section{Discussion}

Resveratrol, known as a natural phytoalexin, is found in some medicinal and edible plants, and shows considerable antibacterial, antioxidant, anti-inflammatory, and anticancer activities [10]. Wang reported that resveratrol can inhibit the growth of Xanthomonas oryzae [11]. However, the potential structure-activity relationships among resveratrol derivatives against Xoo growth and the influence of resveratrol on the metabolic system of Xoo are not known. In this study, the structure-activity relationship and the influence of resveratrol on the metabolic system of Xoo were investigated by ${ }^{1} \mathrm{H}$-NMR-based metabolomics. We found that: (1) The double bond between two benzene rings in resveratrol was essential to inhibit the growth of Xoo; (2) The hydroxyls in these benzene rings might be the main activity group involved in the inhibition of Xoo growth; and (3) resveratrol might cause oxidative stress, as well as disturb energy, nucleic acid, amino acid, and $\mathrm{NAD}^{+}$metabolism, in Xoo cells.

\section{Inhibitory effects on growth of Xoo and the potential structure-activity relationship}

Compounds (1-3) showed strong inhibition activity on the growth of Xoo, with resveratrol demonstrating the 

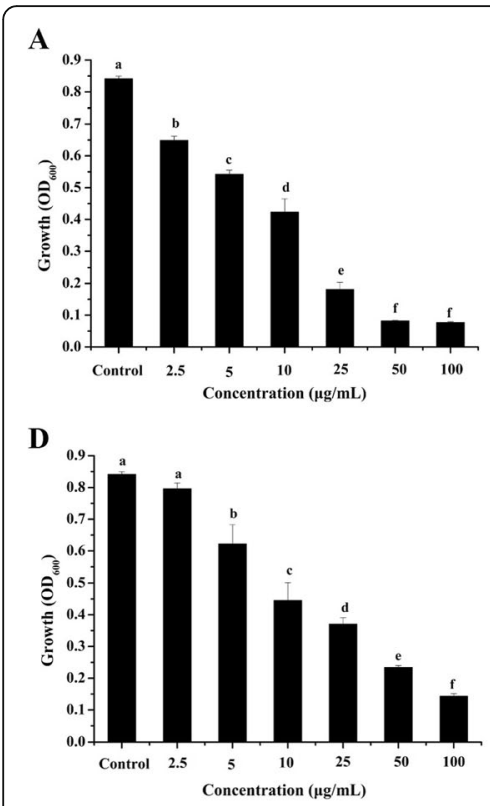

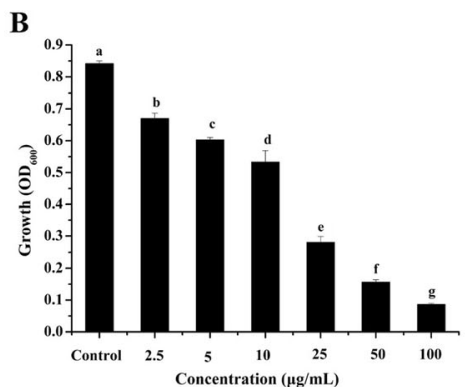

$\mathbf{E}$

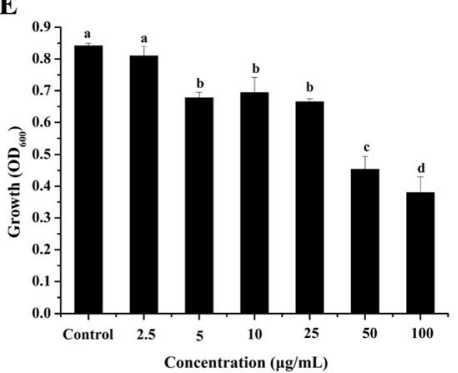

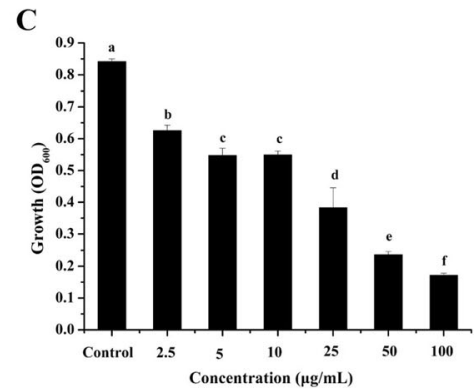

F

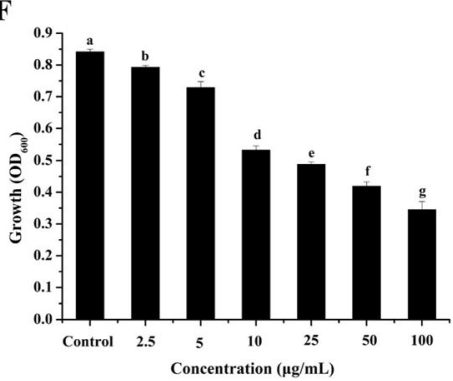

Fig. 2 The growth of Xoo after treated by compounds (1-6), respectively, for 18 h, Compound (1) (a), Compound (2) (b), Compound (3) (c), Compound (4) (d), Compound (5) (e), and Compound (6) (f). Means with different lower-case letters (a, b, c, d) are significantly different ( $p<0.05)$

strongest activity among the tested compounds. Even though resveratrol exhibits antibacterial activity and can inhibit the growth of Xanthomonas [11, 12], the metabolomic mechanisms of the inhibition are still unknown. It was reported that the mechanisms were complex and different for different inhibitors. Difficidin and bacilysin could influence the protein and cell wall synthesis and cell division to inhibit the growth of Xanthomonas [14]. Phenazine-1-carboxylic acid influenced the energy metabolism and disturbed the redox balance in Xoo $[15,16]$. So as a famous antioxidant, resveratrol maybe had effects on the

Table 1 Half maximal inhibitory concentrations $\left(\mathrm{IC}_{50}\right)$ of compounds $\mathbf{1 - 9}$ on the growth of XoO

\begin{tabular}{ll}
\hline Compounds & $\mathrm{IC}_{50}(\mu \mathrm{g} / \mathrm{mL})$ \\
\hline 1 & $11.67 \pm 0.58 \mathrm{c}$ \\
2 & $19.00 \pm 1.00 \mathrm{c}$ \\
3 & $27.00 \pm 3.61 \mathrm{c}$ \\
4 & $36.27 \pm 3.75 \mathrm{~b}$ \\
5 & $123.53 \pm 7.66 \mathrm{a}$ \\
6 & $115.46 \pm 7.93 \mathrm{a}$ \\
7 & nd \\
8 & nd \\
9 & nd
\end{tabular}

\section{nd not detected}

$\mathrm{IC}_{50}$ values were obtained by interpolation from linear regression analysis. Values are presented as mean $\pm \mathrm{SD}(n=3)$, and means in the same column with different lower case letters $(a, b, c)$ are significantly different $(p<0.05)$ energy metabolism and redox balance of Xoo. Subsequently, we investigated the structure-activity relationships among resveratrol and its derivatives. Compared with compounds (1-3), compounds (4-6), which lacked the double bond, showed weaker inhibitory effects on the growth of Xoo. Thus, the double bond in the skeleton of compounds $(\mathbf{1}-\mathbf{3})$ likely played an important role in the inhibition of Xoo growth. Compared with compounds (1-3), all hydroxyls were substituted by methoxyls on the benzene rings in compounds (7-9). Interestingly, compounds (7-9) had no inhibitory effects on Xoo growth, indicating that the hydroxyls were the vital active group for the inhibitory effects on Xoo growth observed for compounds (1-3). For many inhibitors, the double bond and the hydroxyls played important roles in the anti-Xoo activity, such as sphaeropsidin A, the main phytotoxin produced by Diplodia cupressi [17], and it was reported that stilbenoids with at least one free hydroxyl group as a common structural feature suggested an association with the antimicrobial activity, such as against Bacillus subtilis and Pseudomonas syringae [18]. In addition, the literature also showed that the hydroxyls and double bond of stilbenoids were the vital active group for QSI activity, resveratrol showed remarkable QSI activity to C. violaceum and P. aeruginosa [8], and antibacterial activity against $S$. aureus, E. coli O157, S. typhimurium, and $V$. parahemolyticus, but these bioactivities were vanished after the hydroxyl group at position 3 was substituted by a glucosyl group $[19,20]$. Thus, the hydroxyls in these benzene rings and the double bond between two benzene rings in resveratrol were vital for antibacterial activity. 


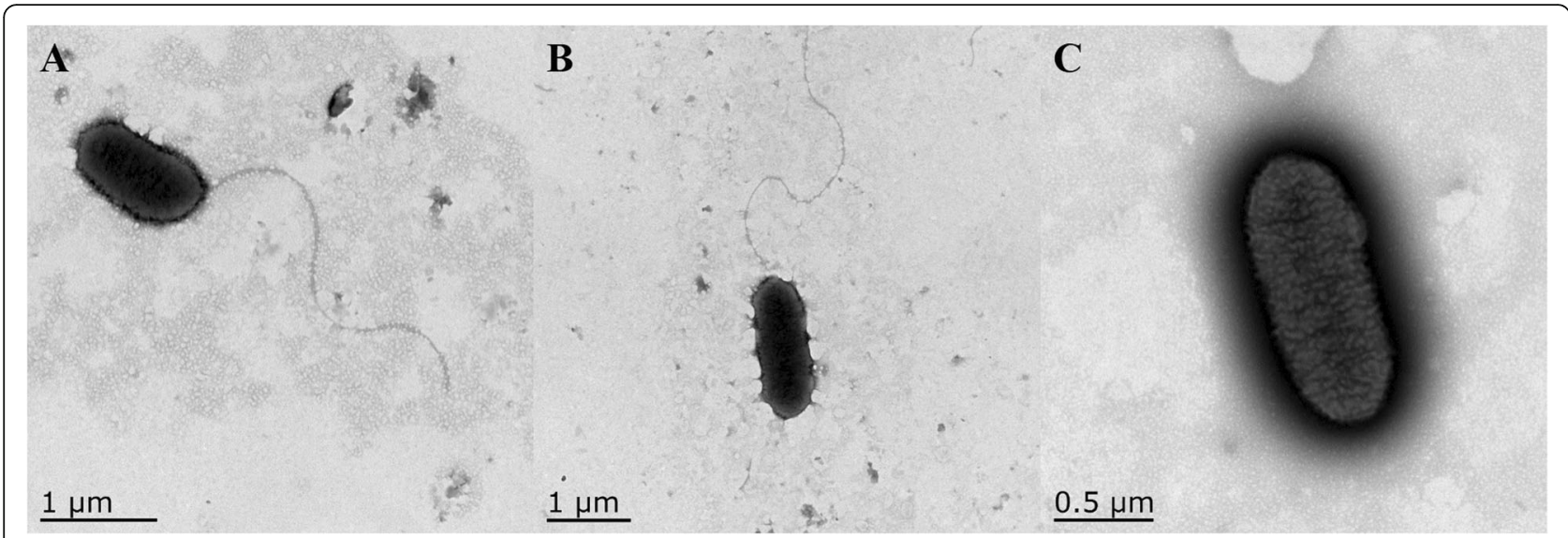

Fig. 3 Effects of resveratrol on flagella of Xoo: blank control (a), DMSO control (b), and resveratrol-treated groups (c)

\section{Effects of resveratrol on the flagella of Xoo}

Flagella are essential for the virulence of organisms such as Pseudomonas and Xanthomonas, and play an important role in the pathogenesis of other mucosal infections as they are directly related to chemotaxis and motility [21]. Comparison of the control and resveratrol-treated groups (Fig. 3) indicated that resveratrol significantly inhibited flagellum growth of Xoo. This lack of flagella could caused efficiency of chemotaxis and motility, resulting in a decrease in virulence and pathogenicity $[21,22]$.

\section{Oxidative stress}

Oxidative stress reflects an imbalance between the generation of reactive oxygen species (ROS) and antioxidant defense, with antioxidative enzymes playing a central role in determining individual risk of developing oxidative stress. The glutathione (GSH) redox system is an important antioxidant defense mechanism, in which glutathione peroxidase (GPx) and glutathione reductase (GR) catalyze the interconversion between reduced glutathione (GSH) and glutathione disulfide (GSSG) [10]. The level of GSH decrease indicated that it was excessive consumption to counteract oxidative stress because of its natural antioxidant in the cells. Additionally, GSH can clear electrophilic exogenous substances and influence detoxification under catalyzing by glutathione S-transferase (GST), which is an adaptive mechanism to reduce toxic effects. After Xoo was treated with resveratrol, the detoxification mechanism may have exacerbated the depletion of GSH, resulting in accelerated synthesis and significantly decreased levels of precursors such as glutamate and glutamine [23].

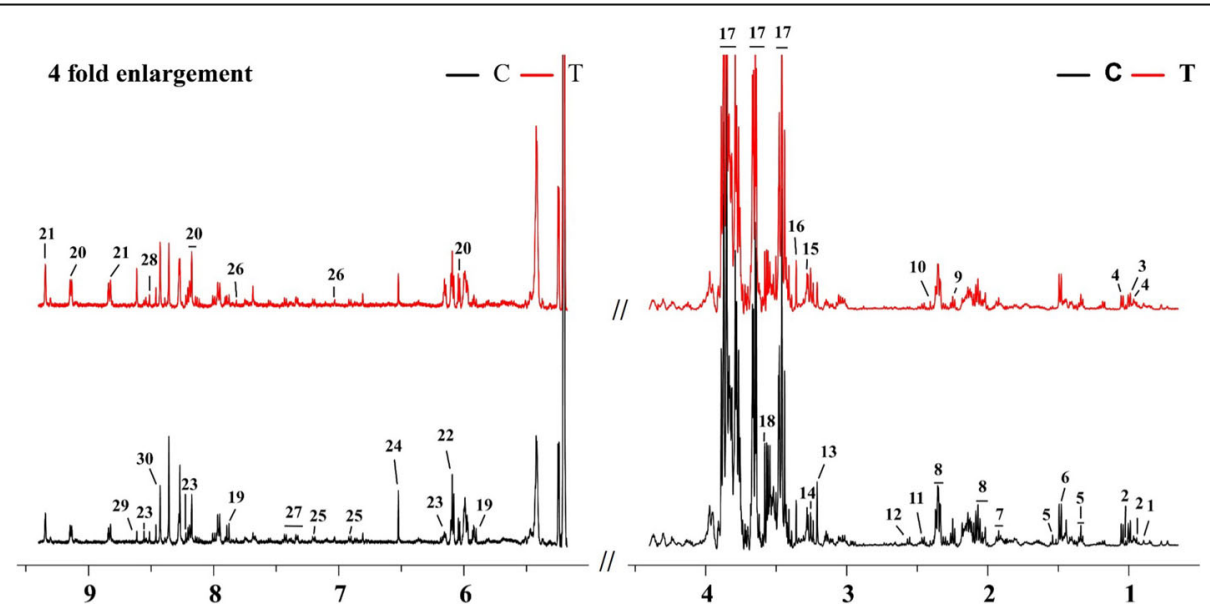

Fig. 4 Typical $500 \mathrm{MHz}$ CPMG ${ }^{1} \mathrm{H}-\mathrm{NMR}$ spectra of Xoo from resveratrol-treated (red line) and control groups (black line): 1, Cholate; 2, Isoleucine; 3 , Leucine; 4, Valine; 5, Suberate; 6, Alanine; 7, Lysine; 8, Glutamate; 9, 2-Aminoadipate; 10, Succinate; 11, Glutamine; 12, Glutathione; 13, Choline; 14, Taurine; 15, Trimethylamine N-oxide; 16, Methanol; 17, Trehalose; 18, Glycine; 19, Uridine; 20, NAD+; 21, NADP+; 22, Inosine; 23, IMP; 24, Fumarate; 25, Tyrosine; 26, Histidine; 27, Phenylalanine; 28, ATP; 29, AMP; 30, Formate 
Table 2 Important Metabolites Assignments in Xoo, Their Fold Change Values, and Associated $p$ Values

\begin{tabular}{|c|c|c|c|c|}
\hline No. & Metabolites & Chemical Shifts (ppm) & $\log _{2}(\mathrm{FC})^{\mathrm{a}}$ & $p^{\mathrm{b}}$ \\
\hline 1 & Cholate & $0.890(\mathrm{~s})$ & 0.316 & $* * *$ \\
\hline 2 & Isoleucine & $0.920(\mathrm{t}), 1.015(\mathrm{~d})$ & 0.224 & \\
\hline 3 & Leucine & $0.950(\mathrm{~d})$ & 0.193 & \\
\hline 4 & Valine & $0.980(\mathrm{~d}), 1.030(\mathrm{~d})$ & -0.189 & $* * *$ \\
\hline 5 & Suberate & $1.320(\mathrm{~m}), 1.515(\mathrm{t})$ & -0.397 & $* * *$ \\
\hline 6 & Alanine & $1.470(\mathrm{~d})$ & -0.060 & \\
\hline 7 & Lysine & $1.860(\mathrm{~m})$ & -0.535 & $* * *$ \\
\hline 8 & Glutamate & $2.010(\mathrm{~m}), 2.325(\mathrm{~m})$ & -0.338 & $* * *$ \\
\hline 9 & 2-Aminoadipate & $2.225(\mathrm{t})$ & -0.636 & $* * *$ \\
\hline 10 & Succinate & $2.400(\mathrm{~s})$ & 0.388 & $*$ \\
\hline 11 & Glutamine & $2.415(\mathrm{~m})$ & -0.464 & $* * *$ \\
\hline 12 & Glutathione & $2.535(\mathrm{~m})$ & -1.055 & $* * *$ \\
\hline 13 & Choline & $3.200(\mathrm{~s})$ & -0.827 & $* * *$ \\
\hline 14 & Taurine & $3.230(t)$ & 0.412 & $* * *$ \\
\hline 15 & Trimethylamine $\mathrm{N}$-oxide & $3.276(\mathrm{~s})$ & 0.077 & \\
\hline 16 & Methanol & $3.350(\mathrm{~s})$ & 0.224 & \\
\hline 17 & Trehalose & $3.435(\mathrm{t}), 3.630(\mathrm{dd}), 3.750(\mathrm{~m})$ & 0.057 & $* *$ \\
\hline 18 & Glycine & $3.565(\mathrm{~s})$ & -0.766 & $* * *$ \\
\hline 19 & Uridine & $5.900(\mathrm{~d}), 7.870(\mathrm{~d})$ & -0.267 & $* * *$ \\
\hline 20 & $\mathrm{NAD}+$ & $6.025(\mathrm{~d}), 8.170(\mathrm{~m}), 9.125(\mathrm{~d})$ & 0.622 & $* * *$ \\
\hline 21 & NADP+ & $8.815(\mathrm{~d}), 9.335(\mathrm{~s})$ & 0.597 & $* * *$ \\
\hline 22 & Inosine & $6.070(\mathrm{~d})$ & 0.005 & \\
\hline 23 & IMP & $6.100(\mathrm{~d}), 8.215(\mathrm{~s}), 8.550(\mathrm{~s})$ & 0.418 & $* * *$ \\
\hline 24 & Fumarate & $6.515(\mathrm{~s})$ & -0.159 & $*$ \\
\hline 25 & Tyrosine & $6.895(\mathrm{~d}), 7.177(\mathrm{~d})$ & 0.085 & \\
\hline 26 & Histidine & $7.030(\mathrm{~s}), 7.815(\mathrm{~s})$ & 0.039 & \\
\hline 27 & Phenylalanine & $7.320(\mathrm{~m})$ & -0.079 & \\
\hline 28 & ATP & $8.510(\mathrm{~s})$ & 0.093 & \\
\hline 29 & AMP & $8.610(\mathrm{~s})$ & 1.442 & $* * *$ \\
\hline 30 & Formate & $8.415(\mathrm{~s})$ & 0.550 & $* * *$ \\
\hline
\end{tabular}

a Color coded according to the fold change value, red represents increased and blue represents decreased concentrations of metabolites b $p$-values corrected by BH (Benjamini Hochberg) methods were calculated based on a parametric Student's t-test or a nonparametric Mann-Whitney test (dependent on the conformity to normal distribution). ${ }^{*} p<0.05,{ }^{* *} p<0.01,{ }^{* * *} p<0.001$

Cell membranes are susceptible to oxidative damage due to unsaturated fatty acids [24]. Choline and phosphocholine are crucial for the structural integrity of cell membranes. In the present study, choline was remarkably decreased in the resveratrol-treated group, indicating that choline was excessively consumed to repair damaged membranes caused by ROS. Because of the strong antioxidant activity and protective effects, taurine showed protective effects against oxidative stress [25]. The increase in taurine indicated its requirement was improving to protect the cell from oxidative stress after treatment with resveratrol [26, 27]. Thus, the decrease in choline and increase in taurine indicated that Xoo underwent severe oxidative damage after treatment with resveratrol. 

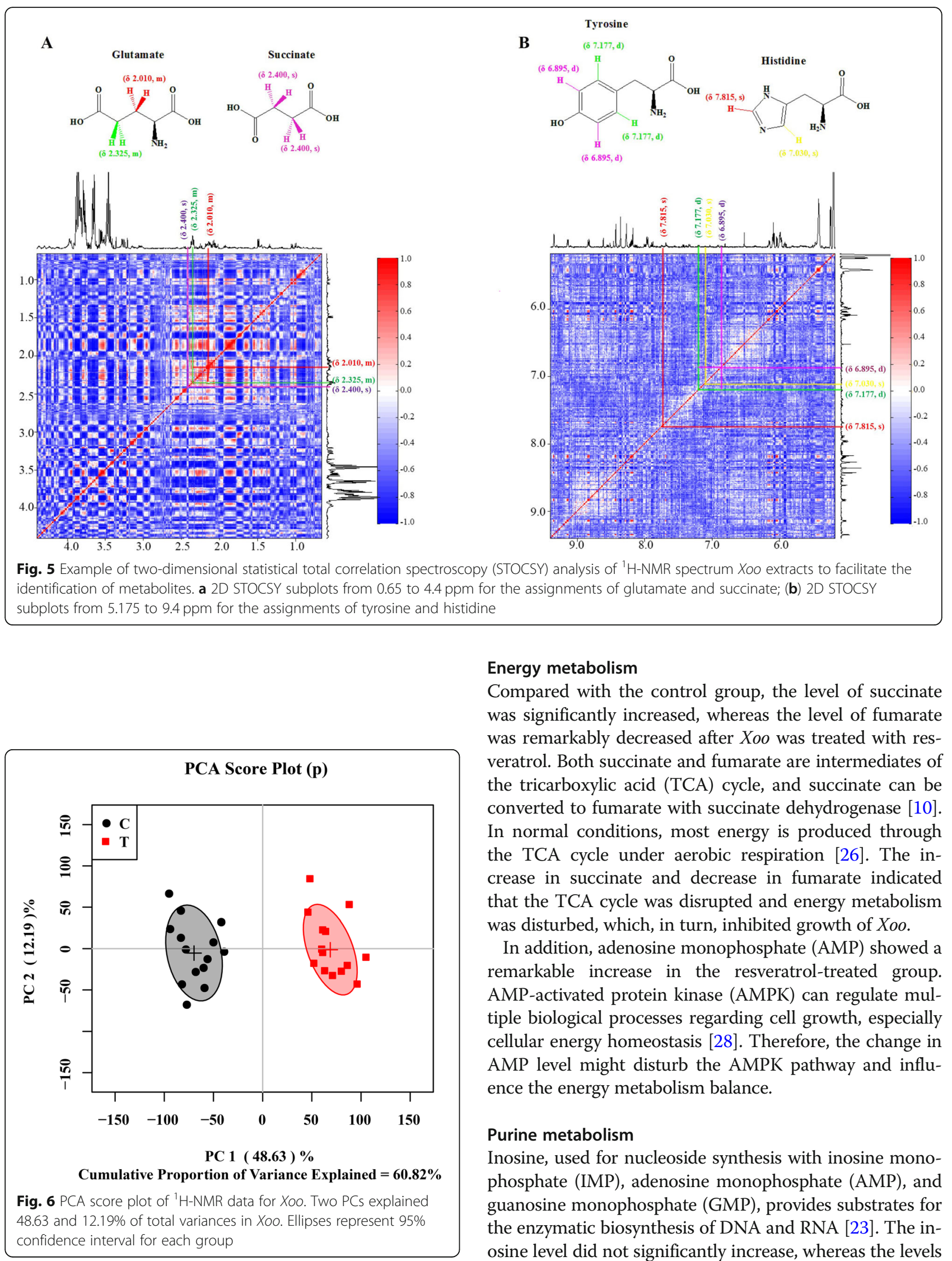

\section{Energy metabolism}

Compared with the control group, the level of succinate was significantly increased, whereas the level of fumarate was remarkably decreased after Xoo was treated with resveratrol. Both succinate and fumarate are intermediates of the tricarboxylic acid (TCA) cycle, and succinate can be converted to fumarate with succinate dehydrogenase [10]. In normal conditions, most energy is produced through the TCA cycle under aerobic respiration [26]. The increase in succinate and decrease in fumarate indicated that the TCA cycle was disrupted and energy metabolism was disturbed, which, in turn, inhibited growth of Xoo.

In addition, adenosine monophosphate (AMP) showed a remarkable increase in the resveratrol-treated group. AMP-activated protein kinase (AMPK) can regulate multiple biological processes regarding cell growth, especially cellular energy homeostasis [28]. Therefore, the change in AMP level might disturb the AMPK pathway and influence the energy metabolism balance.

\section{Purine metabolism}

Inosine, used for nucleoside synthesis with inosine monophosphate (IMP), adenosine monophosphate (AMP), and guanosine monophosphate (GMP), provides substrates for the enzymatic biosynthesis of DNA and RNA [23]. The inosine level did not significantly increase, whereas the levels 

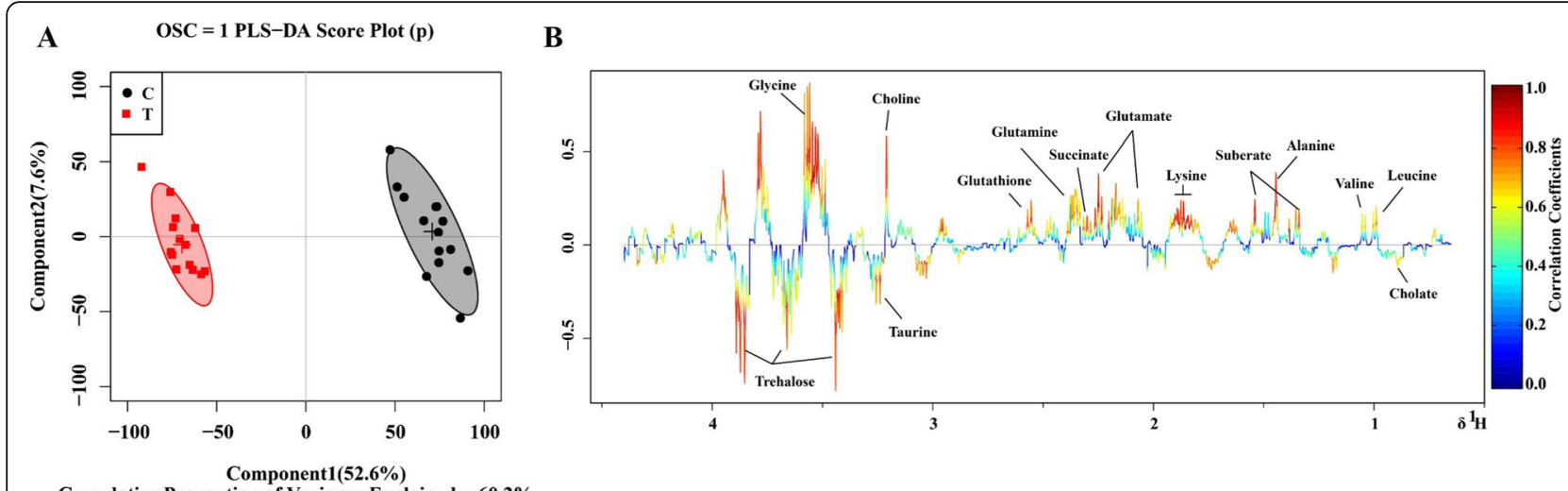

Cumulative Proportion of Variance Explained $=60.2 \%$
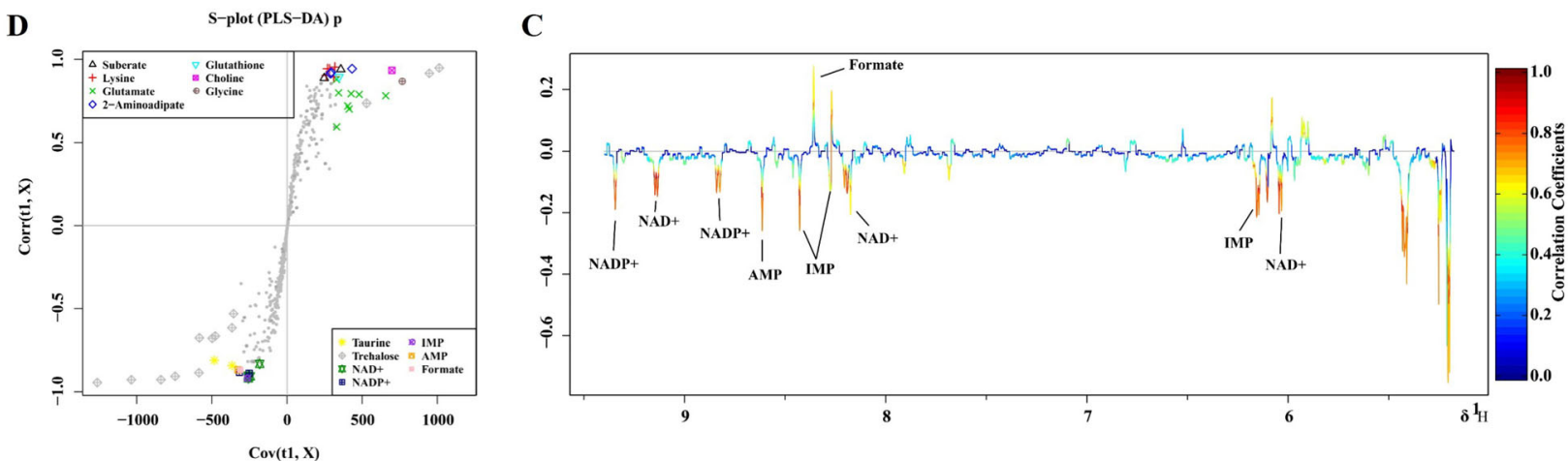

Fig. 7 OSC-PLS-DA analysis of NMR data from Xoo. a Score plot. Component 1 and component 2 explained 60.2\% of total variance in the Xoo sample extracts. b, c Color-coded loadings plots. Color bar was applied, with red and blue representing metabolites that significantly or indistinctively contributed to the separation of groups, respectively. Peaks in positive and negative status reveal decreased and increased metabolites, respectively, relative to the score plot in the resveratrol-treated group. d S-plot

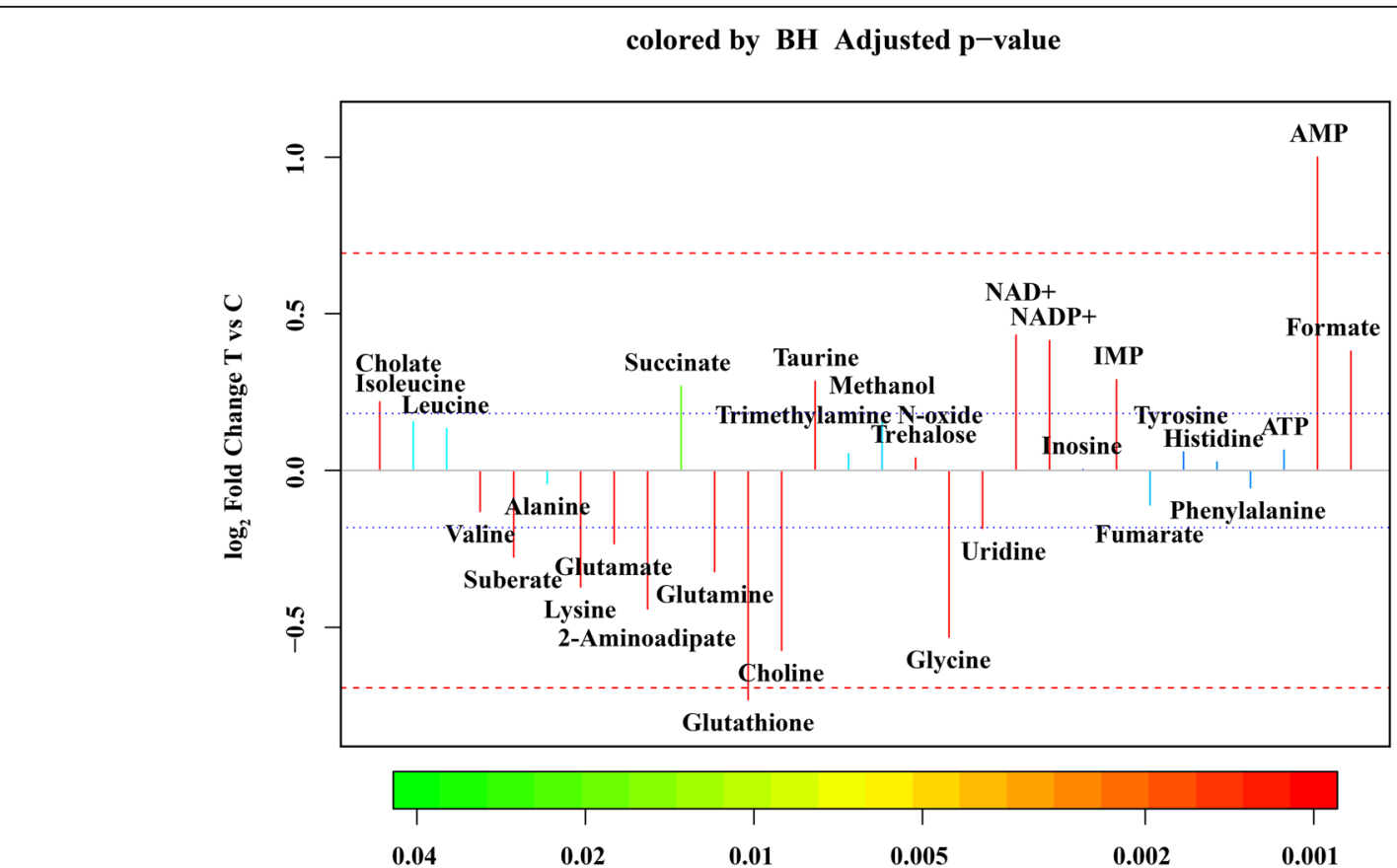

Fig. $\mathbf{8}$ Fold-change plots color coded according to $p$-values adjusted using the Benjamini-Hochberg method, indicating the significance of differences in levels of metabolites in the resveratrol-treated and control groups 
of IMP and AMP did show remarkable increase. ATP hydrolyzes into ADP or AMP to produce energy for the cell, and IMP can convert into AMP by adenylosuccinate lyase [29]. Interestingly, the ATP level did not significantly increase, so the AMP/ATP ratio increased after treatment with resveratrol, indicating that the balance between the production and consumption of ATP was disturbed [30].

\section{Amino acids metabolism}

Valine showed a remarkable decrease in the resveratroltreated group. Valine is a branched-chain amino acid (BCAA, including leucine, isoleucine, and valine) [31]. BCAAs are essential amino acids in vivo and act as vital substrates to regulate protein synthesis [10]. The decrease in valine observed in the present study suggests that the normal protein synthesis of Xoo broke-down due to resveratrol treatment. As proteins are vital substrates for organisms, the growth of Xoo was inhibited after exposure to resveratrol. In addition, some evidence indicates that BCAAs are also nutrient signals that regulate many cellular functions, including cell growth, protein transcription, autophagy, and proliferation [31-33].

Compared with the control group, the level of lysine was significantly decreased in the resveratrol-treated group. Lysine is an essential amino acid and cannot be synthesized with in the cell. Lysine plays a crucial role in the production of carnitine, which can facilitate the oxidization of fatty acids into acetyl CoA, which then enters the TCA cycle. The decrease in lysine in the resveratroltreated group suggests a facilitated conversion to carnitine, which led to the decrease of growing in extreme condition [26].

\section{$\mathrm{NAD}^{+}$metabolism}

The levels of $\mathrm{NAD}^{+}$and $\mathrm{NADP}^{+}$were significantly increased in the resveratrol-treated group, indicating that the metabolism of $\mathrm{NAD}^{+}$was disturbed. $\mathrm{NAD}^{+}$was either de novo synthesized from tryptophan or through the niacin salvage pathway. For the niacin pathway, nicotinamide (NAM) was converted to nicotinamide ribotide (NMN) by nicotinamide phosphoribosyl transferase (Nampt), then $\mathrm{NMN}$ was converted to $\mathrm{NAD}^{+}$by the catalysis of nicotinamide mononucleotide adenylyl transferase (Nmnat) [34]. The significant increase in $\mathrm{NAD}^{+}$observed in the current study indicated that resveratrol enhanced the activity of Nmnat. Through increasing levels of $\mathrm{NAD}^{+}$, Namptmediated $\mathrm{NAD}^{+}$biosynthesis could influence metabolic responses, stress resistance, and cellular differentiation in different cell types [35], and thereby regulate the activity of $\mathrm{NAD}^{+}$-consuming enzymes to improve cellular resistance to damage and stress, and enhance the ability of cells to survive stressful conditions [36]. In conclusion, the increase in $\mathrm{NAD}^{+}$reflects a self-repair mechanism of cells to counteract resveratrol-induced damage.

\section{Conclusion}

Resveratrol showed the strongest inhibition on the growth of Xoo among the nine resveratrol derivatives examined. The potential structure-activity relationship indicated that the double bond of resveratrol contributed to its inhibitory effects on Xoo growth, and the hydroxyls were the vital active group for the inhibitory effects. In addition, resveratrol significantly inhibited Xoo flagella, which might cause chemotaxis and motility deficiency, and thus a decrease in virulence and pathogenicity. The ${ }^{1} \mathrm{H}$ NMR-based metabolomics approach was applied to study the mechanism of resveratrol against Xoo growth. A total of 30 metabolites were identified and assigned. Multivariate statistical analysis highlighted the altered metabolites and indicated that resveratrol could cause oxidative stress and disturb energy, purine, amino acid, and $\mathrm{NAD}^{+}$metabolism in Xoo cells, thus inhibiting Xoo growth. The ${ }^{1} \mathrm{H}$-NMR-based metabolomics approach is a rapid and convenient tool for investigating the mechanism of resveratrol against Xoo growth.

\section{Methods}

\section{Bacterial strains and culture conditions}

The Xanthomonas oryzae pv. oryzae (Xoo) strain PXO99 ${ }^{\mathrm{A}}$ was kindly provided by Prof. G. L. Qian (Nanjing Agricultural University) [37]. Xoo was cultivated at $28^{\circ} \mathrm{C}$ on nutrient agar (NA) medium in plates or in nutrient broth (NB) medium in flasks. NA medium consists of $10 \mathrm{~g}$ of sucrose, $5 \mathrm{~g}$ of peptone, $3 \mathrm{~g}$ of beef extract, $1 \mathrm{~g}$ of yeast powder, and $15 \mathrm{~g}$ of agar powder per liter of distilled water. NB medium contained the same components but lacked agar powder.

\section{Chemicals}

Resveratrol (1) was isolated from Smilax china (purity > 95\%). Oxyresveratrol (2) and piceatannol (3) were purchased from Hangzhou Great Forest Biomedical Ltd. (purity >95\%) (Zhejiang, China). Six derivatives of the above three stilbenoids (1-3), (di-hydro-resveratrol (4), di-hydro-oxyresveratrol (5), di-hydro-piceatannol (6), tri-methyl-resveratrol (7), tetra-methyl-oxyresveratrol (8), and tetra-methyl-piceatannol (9)) were synthesized in our lab. All other chemicals used in this study were purchased from Sigma Chemical (St. Louis, MO, USA).

\section{Minimum inhibitory concentrations (MIC)}

MICs of compounds (1-9) were determined by following the two-fold dilution method [38]. In brief, overnight cultures of Xoo $(1 \%, \mathrm{v} / \mathrm{v})$ were resuspended in fresh nutrient agar (NB) medium in the presence of the samples (0.001-0.1 mg/mL for compounds (1-6) and 0.01-1 mg/ $\mathrm{mL}$ for compounds (7-9)) in 96-well plates, then incubated at $28^{\circ} \mathrm{C}$ and $180 \mathrm{rpm}$ for $18 \mathrm{~h}$. The MICs of compounds (1-9) against Xoo are shown in Table 3. 
Table 3 Minimum inhibitory concentrations (MICs) of the compounds 1-9 against Xoo

\begin{tabular}{ll}
\hline Compounds & MIC $(\mu \mathrm{g} / \mathrm{mL})$ \\
\hline 1 & 1.56 \\
2 & 1.56 \\
3 & 6.25 \\
4 & 3.13 \\
5 & 6.25 \\
6 & 6.25 \\
7 & $>1000$ \\
8 & $>1000$ \\
9 & $>1000$ \\
\hline
\end{tabular}

Antibacterial investigation of compounds (1-9) against Xoo The antibacterial activity of compounds (1-9) against Xoo was investigated as per previous research, with some modifications [39]. Briefly, overnight cultures of Xoo $(1 \%, \mathrm{v} / \mathrm{v})$ were resuspended in the fresh NB medium supplemented with samples at concentration gradients in test tubes, then incubated at $28^{\circ} \mathrm{C}$ and $180 \mathrm{rpm}$ for $18 \mathrm{~h}$. The concentrations of compounds (1-6) were 0 , $2.5,5,10,25,50$, and $100 \mu \mathrm{g} / \mathrm{mL}$, and compounds (7-9) were $0,50,100,200,500$, and $1000 \mu \mathrm{g} / \mathrm{mL}$. The same amount of DMSO was used as the control. Then cultures were analyzed for antibacterial activity at $600 \mathrm{~nm}$ by a spectrophotometer (BioTek, Vermont, USA). Percentage inhibition was calculated as follows:

$$
\text { Inhibition } \%=\left[1-\left(\mathrm{A}_{\mathrm{i}} / \mathrm{A}_{0}\right)\right] \times 100
$$

where $A_{i}$ is the $O_{600}$ of the cultures with compounds (1-9) and $A_{0}$ is the $\mathrm{OD}_{600}$ of the control culture.

\section{Bacterial growth measurement}

The effects of resveratrol on the Xoo growth were measured by following the previous methods, with some modifications [40]. Briefly, overnight culture of Xoo (1\%, $\mathrm{v} / \mathrm{v})$ were resuspended in the fresh NB medium supplemented with resveratrol at different concentrations ( 0 , $2.5,5,10,25,50$, and $100 \mu \mathrm{g} / \mathrm{mL}$ ), then incubated at $28^{\circ} \mathrm{C}$ and $180 \mathrm{rpm}$. The same amount of DMSO was used as the control. The $\mathrm{OD}_{600}$ values of the culture were measured every $2 \mathrm{~h}$ for up to $24 \mathrm{~h}$ by a microplate reader (BioTek, Vermont, USA). The Xoo growth was evaluated by plotting the values of $\mathrm{OD}_{600}$ against time. The results were shown in Fig. S1.

\section{Transmission electron microscope of the Xoo flagella}

Xoo flagella were detected by transmission electron microscopy (TEM) following the methods, with some modifications [22]. Each Xoo overnight culture was diluted into fresh $\mathrm{NB}$ medium at $1 \%(\mathrm{v} / \mathrm{v})$, then incubated at $28^{\circ} \mathrm{C}$ and $180 \mathrm{rpm}$ for $18 \mathrm{~h}$. Resveratrol was added at $11.67 \mu \mathrm{g} / \mathrm{mL}$ to ensure $50 \%$ effect on growth. The suspension was deposited onto grids, then stained with $2 \%$ uranyl acetate for $30 \mathrm{~s}$ and dried for $10 \mathrm{~min}$ at room temperature, with the flagella then observed by TEM (Tecnai 12, Philips, Holland).

\section{Extraction of Xoo metabolite}

Metabolites of Xoo were extracted according to our previous study, with some modifications [10]. Each Xoo overnight culture was diluted into $30 \mathrm{~mL}$ of fresh $\mathrm{NB}$ medium at $1 \%(\mathrm{v} / \mathrm{v})$ in Erlenmeyer flasks, then incubated at $28{ }^{\circ} \mathrm{C}$ and $180 \mathrm{rpm}$ for $18 \mathrm{~h}\left(\mathrm{OD}_{600} \approx 0.82\right)$. Resveratrol was added at $11.67 \mu \mathrm{g} / \mathrm{mL}$ to ensure $50 \%$ effect on growth $\left(\mathrm{OD}_{600} \approx 0.41\right)$. To ensure bacterial equality, two Erlenmeyer flasks of culture were combined to obtain one treated group sample. The same amount of DMSO was used in the control group. Fourteen biological replicates were used for the treatment and control groups, respectively. After incubation, the cell culture was chilled by brief incubation on ice. The cell pellet was obtained by centrifugation at $12,000 \mathrm{rpm}$ for $15 \mathrm{~min}$ at $4{ }^{\circ} \mathrm{C}$. Subsequently, the cell pellet was washed three times with phosphate-buffered saline (PBS), and then transferred to a $10-\mathrm{mL}$ microtube equipped with $3.8 \mathrm{~mL}$ of precooled methanol/water $(1 / 0.9, \mathrm{v} / \mathrm{v})$, and stored at $4{ }^{\circ} \mathrm{C}$ until use. Mixtures were then extracted with a homogenizer for 5 min on ice, with $2 \mathrm{~mL}$ of chloroform added. After vortexing, place the mixtures on ice for $10 \mathrm{~min}$, then centrifuged at $12,000 \mathrm{rpm}$ for $15 \mathrm{~min}$ at $4{ }^{\circ} \mathrm{C}$. Subsequently, the supernatants were transferred to new centrifugal tubes and treated under vacuum with a Speed-Vac Concentrator (Thermo SAVANT, SC110A-230) to completely remove methanol. The supernatants were stored at $-80^{\circ} \mathrm{C}$ overnight, and then lyophilized in a freeze drier. All samples were stored at $-80^{\circ} \mathrm{C}$ for further analysis.

\section{Nuclear magnetic resonance (NMR) measurements}

According to published methods [10], the lyophilized extracts were dissolved in $600 \mu \mathrm{L}$ of $99.8 \% \mathrm{D}_{2} \mathrm{O}$ PBS buffer ( $\mathrm{pH} 7.4$ ) equipped with $0.05 \%(\mathrm{w} / \mathrm{v})$ sodium 3-(trimethylsilyl) propionate-2,2,3,3- $d_{4}$ (TSP). After vortexing, the mixtures were centrifuged at $12000 \mathrm{rpm}$ for $15 \mathrm{~min}$ to discard sediments. The supernatants were transferred to new NMR tubes for ${ }^{1} \mathrm{H}-\mathrm{NMR}$ analysis.

The ${ }^{1} \mathrm{H}$-NMR spectra of samples were recorded on a Bruker AVANCE III $500 \mathrm{MHz}$ NMR spectrometer at $298 \mathrm{~K}$. $\mathrm{D}_{2} \mathrm{O}$ was used for field frequency locking, TSP was used as the chemical shift reference $\left({ }^{1} \mathrm{H}, 0.00 \mathrm{ppm}\right)$. A transverse relaxation-edited Carr-Purcell-Meiboom-Gill

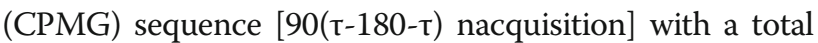
spin-echo delay (2 nt) of $40 \mathrm{~ms}$ was used to suppress the signals of proteins. ${ }^{1} \mathrm{H}-\mathrm{NMR}$ spectra were measured with 128 scans in $32 \mathrm{~K}$ data points with a spectral width of 10 , 
$000 \mathrm{~Hz}$. The spectra were Fourier transformed after multiplying the free induction decays (FIDs) by an exponential weighting function corresponding to a line-broadening of $0.5 \mathrm{~Hz}$.

\section{Data preprocessing and peak assignments}

Before analysis, the ${ }^{1} \mathrm{H}-\mathrm{NMR}$ spectrum was manually phased and baseline corrected using Bruker Topspin 3.0 software (Bruker $\mathrm{GmbH}$, Karlsruhe, Germany) and referenced to TSP at $0.0 \mathrm{ppm}$. Subsequently, the ASCII format files were obtained by the convert of MestReNova (Version 8.0.1, Mestrelab Research SL). And then these files were read into $\mathrm{R}$ software (http://cran.rproject.org) for multivariate analysis. The spectra between 0.2 and $10 \mathrm{ppm}$ were segmented with an average binning of $0.005 \mathrm{ppm}$. The regions influenced by the residual resonance of water was cut off between 4.4 and $5.175 \mathrm{ppm}$. Then, all spectra were conducted probabilistic quotient normalization and mean-centered before multivariate statistical analysis.

The NMR resonances were assigned by querying metabolomics databases, including the Human Metabolome Database (HMDB, http://www.hmdb.ca) and Madison-Qingdao Metabolomics Consortium Database (MMCD, http:// mmcd.nmrfam.wisc.edu), in conjunction with the Chenomx NMR suite 7.5 (Chenomx Inc., Edmonton, Canada) and statistical total correlation spectroscopy (STOCSY) [10].

\section{Multivariate data analysis}

Multivariate statistical analysis was applied to the NMR data, and included principal component analysis (PCA) and supervised orthogonal signal correction partial leastsquares discriminant analysis (OSC-PLS-DA). Unsupervised PCA was first used to reduce the dimensionality of the imported NMR data, and some new latent variables, principal components, were obtained, with such components smaller than variables before transformation. And then filter out irrelevant effects and maximize the discrimination of inter group differences by supervised OSC-PLSDA. The OSC was applied prior to PLS-DA to filter out unrelated variables not concerning class discrimination to minimize the influence of unrelated signals.

The quality of the OSC-PLS-DA model was evaluated by repeated two-fold cross-validation. The $\mathrm{R}^{2}$ and $\mathrm{Q}^{2}$ parameters reflected the prediction ability and the goodness-of-fit of the constructed models. In order to the further validate the supervised model, a permutation test (2000 times) was performed [41]. And color-coded loading plots were constructed to reveal variables that contributed to group separation. The fold-change values of metabolites and their associated $p$-values corrected by the Benjamini and Hochberg-adjusted method were calculated and visualized in colored tables [42]. In addition, receiver operating characteristic (ROC) curves were used to verify the classifier performance of the established OSC-PLS-DA models after 200 times repeated two-fold cross-validation [10].

\section{Univariate statistical analysis}

Univariate analyses, including nonparametric MannWhitney tests and the parametric Student's $t$-tests [10], which were used to detect difference in crucial metabolites between groups. The fold-change values of the identified metabolites as well as $p$-values between groups were calculated. Then false discovery rate was controlled by adjusting p-values according to the Benjamini-Hochberg method when proceeding with multiple comparisons.

\section{Statistical analysis}

All experiments were run in triplicate, and experimental results were expressed as means \pm standard deviation or averages. Data were analyzed by one-way analysis of variance (ANOVA) and Duncan's multiple range test were performed using SPSS version 17.0 (SPSS Inc., Chicago, IL, USA) statistical software with a significant $P$ value of $p<0.05$.

\section{Supplementary information}

Supplementary information accompanies this paper at https://doi.org/10. 1186/s12866-020-01803-W.

Additional file 1: Table S1. ${ }^{13} \mathrm{C}-\mathrm{NMR}(125 \mathrm{MHz})$ spectroscopic data of compounds (4-9). Figure S1. Effects of resveratrol on Xoo growth with different concentrations.

\section{Abbreviations}

Xoo: Xanthomonas oryzae pv. Oryzae; BB: Bacterial blight; QSI: Quorum sensing inhibitor; PCA: Principal component analysis; MIC: Minimum Inhibitory Concentrations; ROS: Reactive oxygen species; GSH: Glutathione; GPx: Glutathione peroxidase; GR: Glutathione reductase; GST: Glutathione Stransferase; TCA: Tricarboxylic acid; AMP: Adenosine monophosphate; AMPK: AMP-activated protein kinase; IMP: Inosine monophosphate; GMP: Guanosine monophosphate; BCAA: Branched-chain amino acid; NAM: Nicotinamide; NMN: Nicotinamide ribotide; Nampt: Nicotinamide phosphoribosyltransferase; Nmnat: Nicotinamide mononucleotide adenylyltransferase; CPMG: Carr-Purcell-Meiboom-Gill; FIDs: Free induction decays; MMCD: Madison-Qingdao Metabolomics Consortium Database; OSCPLS-DA: Orthogonal signal correction partial least-squares discriminant analysis; ROC: Receiver operating characteristic; ANOVA: One-way analysis of variance; NMR: Nuclear Magnetic Resonance

\section{Acknowledgements}

The authors thank Prof. Junsong Wang for his analysis of the metabolome data. The authors thank Prof. Guoliang Qian for the gift of Xanthomonas oryzae pv. oryzae (Xoo) strain PXO99 ${ }^{A}$ (Nanjing Agricultural University).

\section{Authors' contributions}

RY, HL and AJ designed the experiments. GQ, JW and AJ conducted the experiments. $\mathrm{HL}$ and RY performed the experiments and wrote the main manuscript text. $H L$ and RY prepared the Figs. $H L, R Y, Y G, G Q, X Y$ and JW analyzed the data and reviewed the manuscript. All authors have read the manuscript and approved its final version.

\section{Funding}

This study was supported by National Natural Science Foundation of China (41766006), the National Key Research and Development Program of China 
(2017YED0201401), and Six Talent Peaks Project in Jiangsu Province. The parties providing funding were not involved in the study design, data collection and the decision to submit the work for publication.

\section{Availability of data and materials}

All data generated or analysed of this study are described in this paper.

\section{Ethics approval and consent to participate}

Not applicable.

\section{Consent for publication}

Not applicable.

\section{Competing interests}

The authors declare that they have no conflict of interest.

\section{Author details}

'School of Environmental and Biological Engineering, Nanjing University of Science and Technology, Nanjing 210094, China. ${ }^{2}$ Engineering Research Center for Utilization of Tropical Polysaccharide Resources, Ministry of Education, Hainan University, Haikou 570228, China. ${ }^{3}$ Inspection and Pattern Evaluation Department, Suzhou Institute of Metrology, Suzhou 215000, China. ${ }^{4}$ College of Plant Protection, Nanjing Agricultural University, Nanjing 210095, China. ${ }^{5}$ School of Science, Hainan University, Haikou 570228, China.

\section{Received: 29 October 2019 Accepted: 26 April 2020} Published online: 14 May 2020

\section{References}

1. Qian G, Zhou Y, Zhao Y, Song Z, Wang S, Fan J, Hu B, Venturi V, Liu F. Proteomic analysis reveals novel extracellular virulence-associated proteins and functions regulated by the diffusible signal factor (DSF) in Xanthomonas oryzae pv. oryzicola. J Proteome Res. 2013;12(7):3327-41.

2. Shen Y-p, Zou L-f, Li Y-r, Zou H-s, Liu X-I, Chen G-y. Xoryp_08180 of Xanthomonas oryzae pv. oryzicola, Encoding a Hypothetical Protein, is Regulated by HrpG and HrpX and Required for Full Virulence in Rice. J Integr Agr. 2012;11(4):600-610.

3. Tian Y, Zhao Y, Xu R, Liu F, Hu B, Walcott RR. Simultaneous detection of Xanthomonas oryzae pv. oryzae and X. oryzae pv. oryzicola in Rice seed using a padlock probe-based assay. Phytopathology. 2014;104(10):1130-7.

4. Muangham S, Pathom-Aree W, Duangmal K. Melanogenic actinomycetes from rhizosphere soil-antagonistic activity against Xanthomonas oryzae and plant-growth-promoting traits. Can J Microbiol. 2015;61(2):164-70.

5. Xu Y, Zhu X-F, Zhou M-G, Kuang J, Zhang Y, Shang Y, Wang J-X. Status of streptomycin resistance development in Xanthomonas oryzae pv.oryzae and Xanthomonas oryzae pv.oryzicolain China and their resistance characters. J Phytopathol. 2010;158(9):601-8.

6. Khan JA, Siddiq R, Arshad HMI, Anwar HS, Saleem K, Jamil FF. Chemical control of bacterial leaf blight of rice caused by Xanthomonas oryzae pv. oryzae. Pak J Phytopathol. 2012;24(2):97-100.

7. Kagale S, Marimuthu T, Thayumanavan B, Nandakumar R, Samiyappan R. Antimicrobial activity and induction of systemic resistance in rice by leaf extract of Datura metel against Rhizoctonia solani and Xanthomonas oryzae pv. oryzae. Physiol Mol Plant Pathol. 2004;65(2):91-100.

8. Sheng JY, Chen TT, Tan XJ, Chen T, Jia AQ. The quorum-sensing inhibiting effects of stilbenoids and their potential structure-activity relationship. Bioorg Med Chem Lett. 2015;25(22):5217-20.

9. Guiso M, Marra C, Farina A. A new efficient resveratrol synthesis. Tetrahedron Lett. 2002;43(4):597-8.

10. Chen T, Sheng J, Fu Y, Li M, Wang J, Jia AQ. ${ }^{1} H$ NMR-based global metabolic studies of Pseudomonas aeruginosa upon exposure of the quorum sensing inhibitor resveratrol. J Proteome Res. 2017;16(2):824-30.

11. Wang L, Vinogradov EV, Bogdanove AJ. Requirement of the lipopolysaccharide O-chain biosynthesis gene wxocB for type III secretion and virulence of Xanthomonas oryzae pv. oryzicola. J Bacteriol. 2013;195(9): 1959-69.

12. Yu SM, Lee HK, Jeong US, Baek SH, Noh TH, Kwon SJ, Yong HL. Inhibitory effects of resveratrol and Piceid against pathogens of Rice Plant, and disease resistance assay of transgenic Rice Plant transformed with Stilbene synthase gene. Res Plant Dis. 2013;19(3):177-82.
13. Tian F, Yu C, Li H, Wu X, Li B, Chen H, Wu M, He C. Alternative sigma factor RpoN2 is required for flagellar motility and full virulence of Xanthomonas oryzae pv. oryzae. Microbiol Res. 2015;170:177-83.

14. Wu L, Wu H, Chen L, Yu X, Borriss R, Gao X. Difficidin and bacilysin from Bacillus amyloliquefaciens FZB42 have antibacterial activity against Xanthomonas oryzae rice pathogens. Sci Rep. 2015;5:12975.

15. Xu S, Luo J, Pan X, Liang X, Wu J, Zheng W, Chen C, Hou Y, Ma H, Zhou M. Proteome analysis of the plant-pathogenic bacterium Xanthomonas oryzae pv. oryzae. Biochim Biophys Acta. 2013;1834(8):1660-70.

16. Xu S, Pan X, Luo J, Wu J, Zhou Z, Liang X, He Y, Zhou M. Effects of phenazine-1-carboxylic acid on the biology of the plant-pathogenic bacterium Xanthomonas oryzae pv. oryzae. Pestic Biochem Physiol. 2015; 117:39-46.

17. Evidente A, Venturi V, Masi M, Degrassi G, Cimmino A, Maddau L, Andolfi A. In vitro antibacterial activity of sphaeropsidins and chemical derivatives toward Xanthomonas oryzae pv. oryzae, the causal agent of rice bacterial blight. J Nat Prod. 2011;74(12):2520-5.

18. Aslam SN, Stevenson PC, Kokubun T, Hall DR. Antibacterial and antifungal activity of cicerfuran and related 2-arylbenzofurans and stilbenes. Microbiol Res. 2009;164(2):191-5

19. Xie Y, Chen J, Xiao A, Liu L. Antibacterial activity of polyphenols: structureactivity relationship and influence of hyperglycemic condition. Molecules. 2017;22(11):1913.

20. Nawaz W, Zhou Z, Deng S, Ma X, Ma X, Li C, Shu X. Therapeutic versatility of resveratrol derivatives. Nutrients. 2017;9(11):1188.

21. Chen L, Hu B, Qian G, Wang C, Yang W, Han Z, Liu F. Identification and molecular characterization of twin-arginine translocation system (tat) in Xanthomonas oryzae pv. oryzae strain PXO99. Arch Microbiol. 2009;191(2): 163-70.

22. Yu C, Chen H, Tian F, Yang F, Yuan X, Yang CH, He C. A ten genecontaining genomic island determines flagellin glycosylation: implication for its regulatory role in motility and virulence of Xanthomonas oryzae $p v$. oryzae. Mol Plant Pathol. 2018;19(3):579-92.

23. Chen T, Liu Y, Li MH, Xu HD, Sheng JY, Zhang L, Wang JS. Integrated ${ }^{1} H$ NMR-based metabolomics analysis of earthworm responses to sub-lethal $\mathrm{Pb}$ exposure. Environ Chem. 2016;13(5):792-803.

24. Schmidley JW. Free radicals in central nervous system ischemia. Stroke. 1990;21(7):1086-90.

25. Hagar $\mathrm{HH}$. The protective effect of taurine against cyclosporine A-induced oxidative stress and hepatotoxicity in rats. Toxicol Lett. 2004;151(2):335-43.

26. Li M-H, Ruan L-Y, Liu Y, Xu H-D, Chen T, Fu Y-H, Jiang L, Wang J-S. Insight into biological system responses in goldfish (Carassius auratus) to multiple doses of avermectin exposure by integrated ${ }^{1} \mathrm{H}$ NMR-based metabolomics. Toxicol Res. 2015;4(5):1374-88.

27. Li M, Wang J, Lu Z, Wei D, Yang M, Kong L. NMR-based metabolomics approach to study the toxicity of lambda-cyhalothrin to goldfish (Carassius auratus). Aquat Toxicol. 2014;146:82-92.

28. Swan $\mathrm{CL}$, Sistonen L. Cellular stress response cross talk maintains protein and energy homeostasis. EMBO J. 2015;34(3):267-9.

29. Kappock TJ, Ealick SE, Stubbe J. Modular evolution of the purine biosynthetic pathway. Curr Opin Chem Biol. 2000;4(5):567-72.

30. Jiang $L$, Si ZH, Li MH, Zhao H, Fu YH, Xing YX, Hong W, Ruan LY, Li PM, Wang JS. ${ }^{1} \mathrm{H}$ NMR-based metabolomics study of liver damage induced by ginkgolic acid (15:1) in mice. J Pharmaceut Biome. 2017;136:44-54.

31. Li MH, Du HZ, Kong GJ, Liu LB, Li XX, Lin SS, Jia AQ, Yuan ST, Sun L, Wang JS. Nuclear magnetic resonance-based metabolomics approach to evaluate the prevention effect of Camellia nitidissima chi on colitis-associated carcinogenesis. Front Pharmacol. 2017;8:447.

32. Nicklin $P$, Bergman $P$, Zhang $B$, Triantafellow $E$, Wang $H$, Nyfeler $B$, Yang $H$ Hild M, Kung C, Wilson C. Bidirectional transport of amino acids regulates mTOR and autophagy. Cell. 2009;136(3):521-34.

33. Waickman AT, Powell JD. mTOR, metabolism, and the regulation of T-cell differentiation and function. Immunol Rev. 2012;249(1):43-58.

34. Zhao H, Si Z-H, Li M-H, Jiang L, Fu Y-H, Xing Y-X, Hong W, Ruan L-Y, Li P-M, Wang J-S. Pyrazinamide-induced hepatotoxicity and gender differences in rats as revealed by a ${ }^{1} \mathrm{H}$ NMR based metabolomics approach. Toxicol Res. 2017;6(1):17-29.

35. Garten A, Petzold S, Körner A, Imai S, Kiess W. Nampt: linking NAD biology, metabolism and cancer. Trends Endocrin Met. 2009;20(3):130-8.

36. Rongvaux A, Galli M, Denanglaire S, Van GF, Drèze PL, Szpirer C, Bureau F, Andris F, Leo O. Nicotinamide phosphoribosyl transferase/pre-B cell colony- 
enhancing factor/visfatin is required for lymphocyte development and cellular resistance to genotoxic stress. J Immunol. 2008;181(7):4685-95.

37. Wang B, Wu GC, Zhang YQ, Qian GL, Liu FQ. Dissecting the virulencerelated functionality and cellular transcription mechanism of a conserved hypothetical protein in Xanthomonas oryzae pv. Oryzae. Mol Plant Pathol. 2018;19(8):1859-72.

38. Zhou J, Bi S, Chen H, Chen T, Yang R, Li M, Fu Y, Jia AQ. Anti-biofilm and Antivirulence activities of metabolites from Plectosphaerella cucumerina against Pseudomonas aeruginosa. Front Microbiol. 2017;8:769.

39. Chen J, Wang $X$, Han H. A new function of graphene oxide emerges: inactivating phytopathogenic bacterium Xanthomonas oryzae pv. Oryzae. J Nanopart Res. 2013;15(5):1658.

40. Yang R, Guan Y, Zhou J, Sun B, Wang Z, Chen H, He Z, Jia A.

Phytochemicals from Camellia nitidissima chi flowers reduce the Pyocyanin production and motility of Pseudomonas aeruginosa PAO1. Front Microbiol. 2017:8:2640.

41. Xia J, Psychogios N, Young N, Wishart DS. MetaboAnalyst: a web server for metabolomic data analysis and interpretation. Nucleic Acids Res. 2009 37(Web Server issue):W652-W660.

42. Benjamini Y, Hochberg Y. Controlling the false discovery rate - a practical and powerful approach to multiple testing. J Roy Stat Soc Ser B. 1995;57(1):289-300.

\section{Publisher's Note}

Springer Nature remains neutral with regard to jurisdictional claims in published maps and institutional affiliations.

Ready to submit your research? Choose BMC and benefit from:

- fast, convenient online submission

- thorough peer review by experienced researchers in your field

- rapid publication on acceptance

- support for research data, including large and complex data types

- gold Open Access which fosters wider collaboration and increased citations

- maximum visibility for your research: over $100 \mathrm{M}$ website views per year

At BMC, research is always in progress.

Learn more biomedcentral.com/submissions 